\title{
Design of a High Resolution Probe Head for Electromagnetic Turbulence Investigations in W7-X
}

\author{
P. Agostinetti, M. Spolaore, M. Brombin, V. Cervaro, L. Franchin, O. Grulke, C. Killer, \\ E. Martines, M. Moresco, S. Peruzzo, N. Vianello, M. Visentin
}

\begin{abstract}
An insertable probe head called High Resolution Probe (HRP) has been developed at Consorzio RFX in the framework of the EUROfusion S1 work program and in collaboration with IPP Greifswald, to study the electrostatic and electromagnetic features of turbulence in the edge region of Wendelstein 7-X using the Multi-Purpose Manipulator (MPM). The paper reports the design development of the HRP diagnostic head, from the choice of the sensors to the engineering design. The assumptions and evaluations supporting the main design choices, together with the R\&D tests carried out to check the most critical parts, are described in detail.
\end{abstract}

Index Terms-insertable probe, electromagnetic fluctuations, stellarator, W7-X

\section{INTRODUCTION}

W ENDELSTEIN 7-X (W7-X) is a large, superconducting stellarator with modular coils and an optimized magnetic field [1][2].

A multi-purpose manipulator (MPM) system has been developed and installed on the $\mathrm{W} 7-\mathrm{X}$ vessel to investigate the edge plasmas of the stellarator [3][4]. It is a flexible tool for integration of a variety of different diagnostics as e. g. electrical probes, magnetic coils, material collection, or material exposition probes, and gas injection [5][6]. The system is designed as user facility for many diagnostics, which can be mounted on a unique interface without affecting the W7$\mathrm{X}$ vacuum. The manipulator system, located in the equatorial plane, transports the inserted diagnostic probe to the edge of the inner vacuum vessel. From there the probe can be moved over a maximum distance of $350 \mathrm{~mm}$ to different positions inside the plasma with a maximum acceleration and deceleration of $30 \mathrm{~m} \mathrm{~s}^{-2}$.

In the framework of the EUROfusion S1 work program for the preparation and exploitation of W7-X campaigns, an insertable probe head called HRP (High Resolution Probe) was developed by Consorzio RFX in collaboration with IPP Greifswald, to investigate the electrostatic and electromagnetic features of turbulence in the edge region of W7-X using the MPM.

This paper reports the design development of the HRP head, from the choice of the sensors to the engineering design. The assumptions and evaluations supporting the main design choices, together with the R\&D tests carried out to check the most critical parts, are described in detail.

\section{Conceptual Design}

The principal aim of the HRP head is to provide information on parallel current density associated to L-mode filamentary

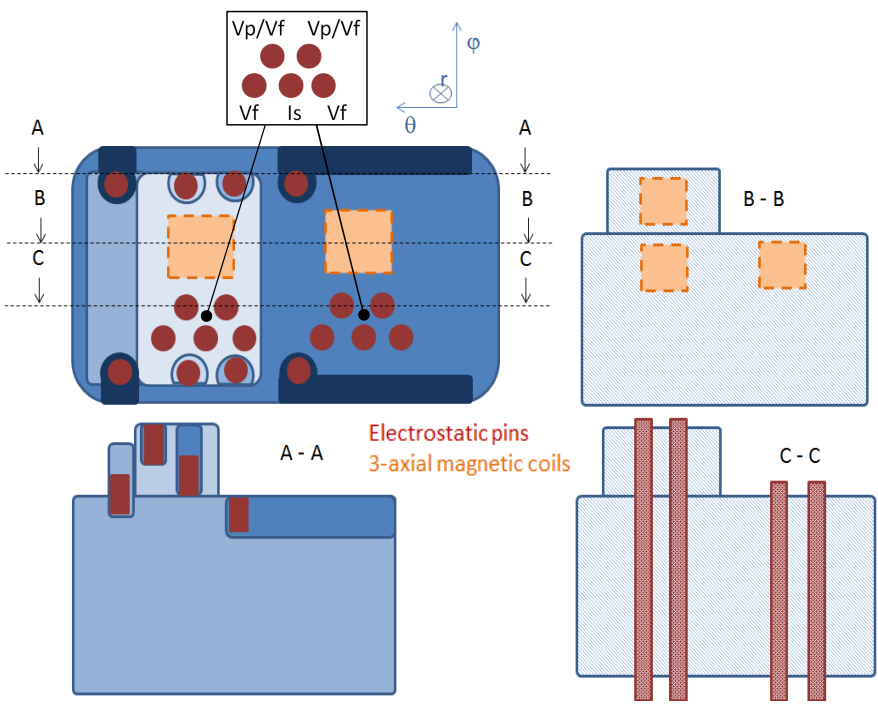

Fig. 1. Conceptual design of the High Resolution Probe Head. Overall

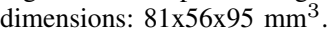

turbulent structures as well as on ELMy structures in H-mode $[7][8][9][10][11]$. Furthermore the possibility to measure the time evolution of radial profiles of flow was considered as a further interesting part of the study, given the strong interplay expected between the turbulent fluctuation and the average flows.

The conceptual probe head layout is shown in Fig. 1. The front side view is shown on the top-left of the figure. The electrostatic pins are shown in dark red, while the magnetic coils are represented by the orange squares. The probe head case is represented by the blue structure. As a matter of comparison the magnetic 3-axial coil occupies a volume of approximately $1 \mathrm{~cm}^{3}$. The probe head is conceived according to the reference frame shown in Fig. 1, with the long edge parallel to the poloidal direction $(\theta)$ and the short edge parallel to the toroidal direction $(\varphi)$. The probe head case structure follows the concept of a "castellated shape", in order to have electrostatic pin measurements in different radial positions, with a limited shadow effect due to the flux tube related to the probe head structure itself.

In Fig. 1 this concept is evidenced by the different blue shades, that in the front side view refers to poloidal-toroidal planes, placed from inner radial position (lighter blue) to relatively outer radial position (darker blue). As seen in the front side view, two groups of 5-pins are distinguishable 

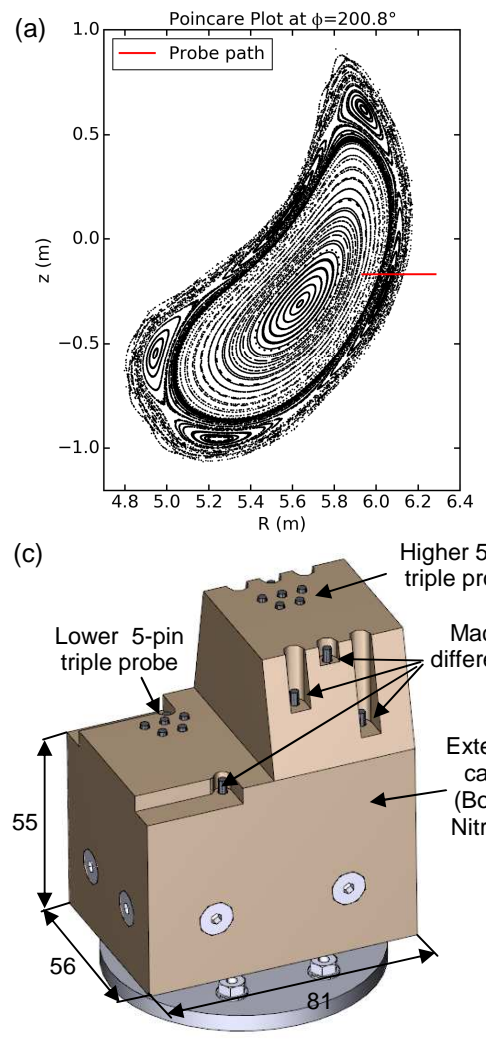

(b)

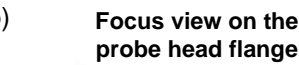

Interface surface with HRP head

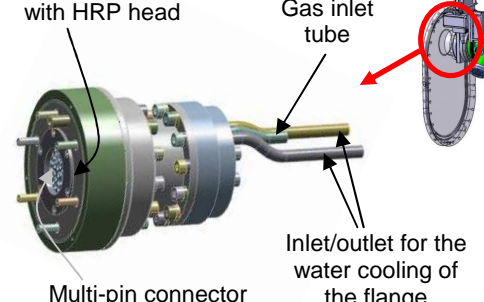

Multi-pin connector the flange

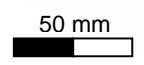

(d)

the
Overview of the Multi-Purpose
Manipulator (MPM)

\section{,} Groch probes at four Mach probes at four
different radial positions External case Nitride)

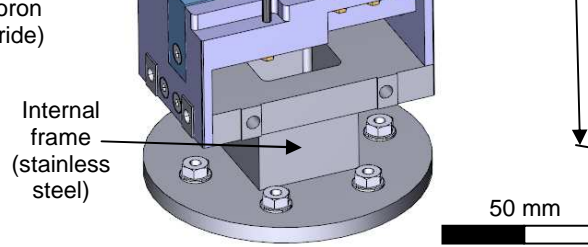

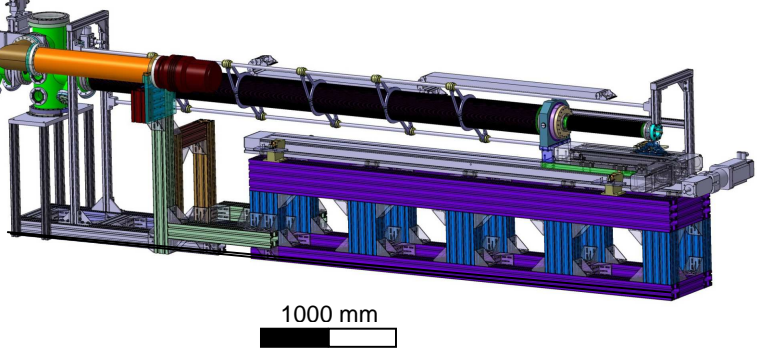

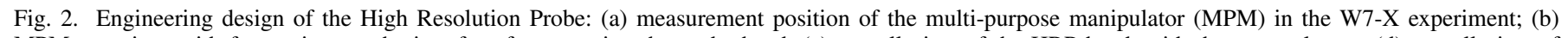

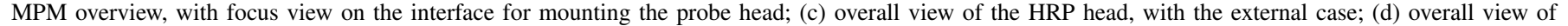
the HRP head, without the external case; (e) section view showing the connections of the pin probes; (f) detail view of a pin probe.

and placed at two different radial positions. These groups are conceived to provide the possibility to realize the 5-pin balanced triple probe configuration [12] as indicated in the upper box of Fig. 1, where the ion saturation current, $I_{s}$, the floating potential, $\mathrm{V}_{f}$, the positively biased potential $V_{p}$, are indicated. The method allows measurements of electron temperature, poloidal electric field, electron density, virtually in the same location, so that also the measurement of the electrostatic particle flux can also be estimated. On the other side different combination of floating potential measurements allow the estimate of $E \times B$ flow.

In addition the probe head is equipped with four pairs of pins arranged in order to provide four Mach probes placed at different radial positions, and with three 3-axial magnetic probes. The latter ones are arranged to form an L-shaped configuration lying on the cross-field plane (radial-poloidal in the Fig. 1). The concept allows for a direct estimate of the parallel current density fluctuations from the Ampere's law.

This method represents a simplified 2D version of the one adopted in the Cluster mission for the measurement of currents in the magnetosphere [13] and it was applied for the first time in a fusion experiment in RFX-mod, with a probe head combining magnetic and electrostatic pins [7]. By using the analogous diagnostic concept the estimate of the current density associated to turbulent filaments was estimated and compared in different experiments including RFX-mod in Reversed Field Pinch as well as in tokamak configuration, in Torpex device, in the stellarator TJ-II [14] and also in COMPASS tokamak [15], where a direct estimate of current density inside ELMs was provided [16].

Summarizing the proposed sensors to be installed on the HRP head are the following:

- two 5-pin triple probes at two radial positions, providing measurements of electron temperature, plasma density, electric fields, plasma flow and particle flux;

- four Mach probes at four different radial positions, for radial profile flow measurements;

- three 3-axial magnetic probes on $(r, \theta)$ plane, measuring the fluctuations of the parallel current density.

\section{ENGINEERING DESIGN}

The guideline for the design of the various components of the HRP head has been the combination of the maximum reliability with a manufacturing and assembly processes maintained as easy as possible. To do this, the manufacturing feasibility of the various components has been considered since the beginning of the design phase, by a close interaction among the drawing office and the mechanical workshop of Consorzio RFX. During all the design phases, the mechanical and thermal robustness of the head has been taken in great consideration, by allocating a sufficient thickness for the most critical parts. On this regard, the most critical component is the external ceramic case, that has been designed with a minimum thickness of $5 \mathrm{~mm}$. 
The measurement point of the MPM in the W7-X experiment is shown in Fig. 2a. The MPM features a mounting flange made of stainless steel with 6 fixing bolts, a multi-pin connector and a gas feeding connection [3], as shown in Fig. $2 b$.

The structure of the HRP head was designed to be interfaced with the fixing bolts and the multi-pin connectors of the MPM. It basically consists of an internal frame, made of stainless steel, supporting an external case (shown in Fig. 2c), and a set of probes (electrostatic pins and magnetic sensors) supported by a vespel structure (shown in Fig. 2d). The volume of the HRP head has been minimized in order to perform the measurements in a volume limited as much as possible.

The electrostatic pins are inserted from below and then blocked with a stainless steel threaded spacer, as shown in the section views of Fig. 2e and Fig. 2f. A good electrical contact between the pin and the threaded spacer is provided by a spring washer located between the two parts. For an easier manufacturing, the length of the graphite pins has been minimized to $8.5 \mathrm{~mm}$ for the 5-pin triple probes and to 11.5 $\mathrm{mm}$ for the Mach probes. The support system is designed to be compatible also with the large accelerations, up to $30 \mathrm{~m}$ $\mathrm{s}^{-2}$, foreseen during the operations of the HRP head.

The parts facing the plasma are the electrostatic pins and the external case. The main proposed materials are graphite for the electrostatic pins and Boron Nitride (BN) [17] for the external case. While the graphite can be accepted as plasma facing material without specific tests, the possibility to use the Boron Nitride for the plasma facing external case was examined in detail. In fact, the heating system of W7-X experiment is based on Electron Cyclotron Resonant Heating (ECRH) working at a frequency of $140 \mathrm{GHz}$. In order to minimize possible spurious effects that may be caused by conducting materials on the 3axial magnetic sensors measurements, a careful choice of the plasma facing material and in particular for the external case must be made. Among the available $\mathrm{BN}$ on the market, the Combat BN AX05 was selected as the most suitable material, as it is transparent to microwave energy and it provides high resistivity and dielectric strength with a low loss tangent and dielectric constant.

\section{DESIGN OF A SHIELD FOR THE MAGNETIC SENSORS}

It has to be considered that the experimental campaign in W7-X will be characterized by ECRH heating system with a power of $7 \mathrm{MW}$ at the frequency $f_{E C R H}=140 \mathrm{GHz}$. The discharge lengths will eventually approach 10-20 s. In these conditions the definition of the ECRH stray radiation is $P_{E C R H-s t r a y}=50 \mathrm{~kW} / \mathrm{m}^{2}[18]$.

The magnetic pick-up coil sensors constitute an important part of the HRP head design and a specific attention is devoted to this issue. It could be considered that the probe head will be installed on the MPM and most of the time the probe head will be located on the "park position", behind the first wall elements, where a reduction of $25 \%$ on the radiation power can be expected and a further reduction due to the relative distance of ECRH launchers [18].

However the value of $P_{E C R H-s t r a y}=50 \mathrm{~kW} / \mathrm{m}^{2}$ is kept conservatively as a reference for the HRP head design. It
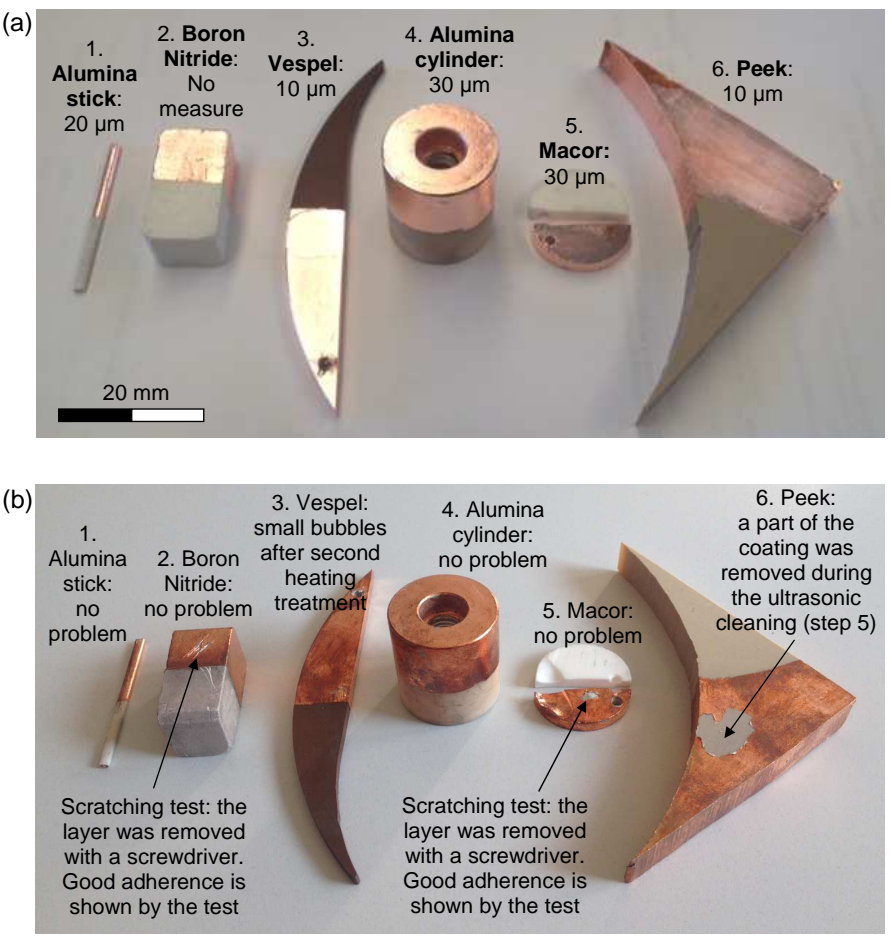

Fig. 3. Thin layer electrodeposition test on different materials: (a) samples after electrodeposition (step 3) with measurement of the electrodeposited copper layer; (b) samples after all tests.

is worth mentioning that if on one side the $\mathrm{BN}$ insulating probe head case can be considered substantially transparent to the $f=140 \mathrm{GHz} \mathrm{ECRH}$ stray radiation, on the other side the magnetic sensors need to be protected from the same radiation. Filtering out the $f=140 \mathrm{GHz}$ however has to be achieved without compromising the magnetic fluctuations to be measured by the sensors. It was evaluated that a good compromise for the HRP head turbulence measurements would be to make the magnetic sensor fluctuations available in the range $f_{\text {measure }} \leq 1 \mathrm{MHz}$.

The suitable shield for magnetic sensor therefore should work as a low-pass filter for the magnetic fluctuation to be measured (up to $f_{\text {measure }}=1 M H z$ ), and at the same time should act as effective shield at $f=140 \mathrm{GHz}$ to prevent the ECRH stray radiation damage of the sensor itself. According to the theory [19], this shield can be obtained by surrounding the magnetic sensors with a very small metallic layer $(5 \mu \mathrm{m})$.

\section{R\&D ON THIN COPPER ELECTRODEPOSITION}

Consorzio RFX has a long experience on copper electrodeposition [20][21], hence to provide the electromagnetic shield it was chosen to test the electrodeposition of a thin copper layer on a non-conductive base material. The base material is required to be electrically insulating, vacuum compatible, providing good adherence for the copper film and having a good resistance to high temperature, at least to $250{ }^{\circ} \mathrm{C}$. In fact, when the coating layer absorbs the ECRH radiation, it can heat up and hence also the base material on which it is connected can reach high temperatures. 
To choose the most suitable base material, six samples with different shapes and materials were compared, as shown in Fig. 3a. The materials tested were Alumina, Boron Nitride, Vespel, Macor and Peek. The main steps of the test were the following:

1) Measurement of the thickness of the sample before the electrodeposition, using a micrometer.

2) Pre-treatment: application of a silver paint on about half of the sample surface.

3) Electrodeposition for 15 minutes with tension of $1 \mathrm{~V}$. All samples were treated together in the same bath.

4) Measurement of the thickness of the sample with the electrodeposited layer, using a micrometer, and evaluation of the electrodeposited layer thickness.

5) Ultrasonic cleaning of the samples in an ethanol bath.

6) Thermal treatment for 1 hour at $120^{\circ} \mathrm{C}$ in vacuum $\left(10^{-6}\right.$ mbar).

7) Drying with nitrogen.

8) Visual check of the status of the electrodeposited layer.

9) Thermal treatment for 1 hour at $250{ }^{\circ} \mathrm{C}$ in vacuum $\left(10^{-6}\right.$ mbar). Note: the Peek sample has been excluded from this treatment because of the low thermal resistance of the material.

10) Drying with nitrogen.

11) Scratching test with a screwdriver on samples 2 (Boron Nitride) and 5 (Macor).

12) Visual check of the status of the electrodeposited layer.

The samples after the electrodeposition (step 4) are shown in Fig. 3a, with indication of the measured thickness of the electrodeposited layers. It can be observed that:

- The electrodeposited layer thickness is different in the different samples. In fact, it was found to depend on the shape of the samples and on the porosity of the base material.

- The values of the electrodeposited layer thickness are generally higher than the required values of $5 \mu \mathrm{m}$. For this reason, the electrodeposition time has been reduced in the manufacturing of the probe sample, described in the following section.

- The coating layer thickness could not be measured for sample 2, because in this case the material increased its dimensions during the galvanic process because it absorbed some liquid.

The samples after all the tests are shown in Fig. 3b. It can be observed that:

- Samples 1 (Alumina stick), 2 (Boron Nitride), 4 (Alumina cylinder) and 5 (Macor) had no problem after all the tests;

- Samples 3 (Vespel) showed some small bubbles after the second heating treatment (step 9);

- In samples 6 (Peek), a part of the coating was detached during the ultrasonic cleaning (step 5).

A scratching test was carried out on samples 2 (Boron Nitride) and 5 (Macor). In both cases, the result was the following:

- light scratching (with a nail) did not cause any damage to the electrodeposited layer;
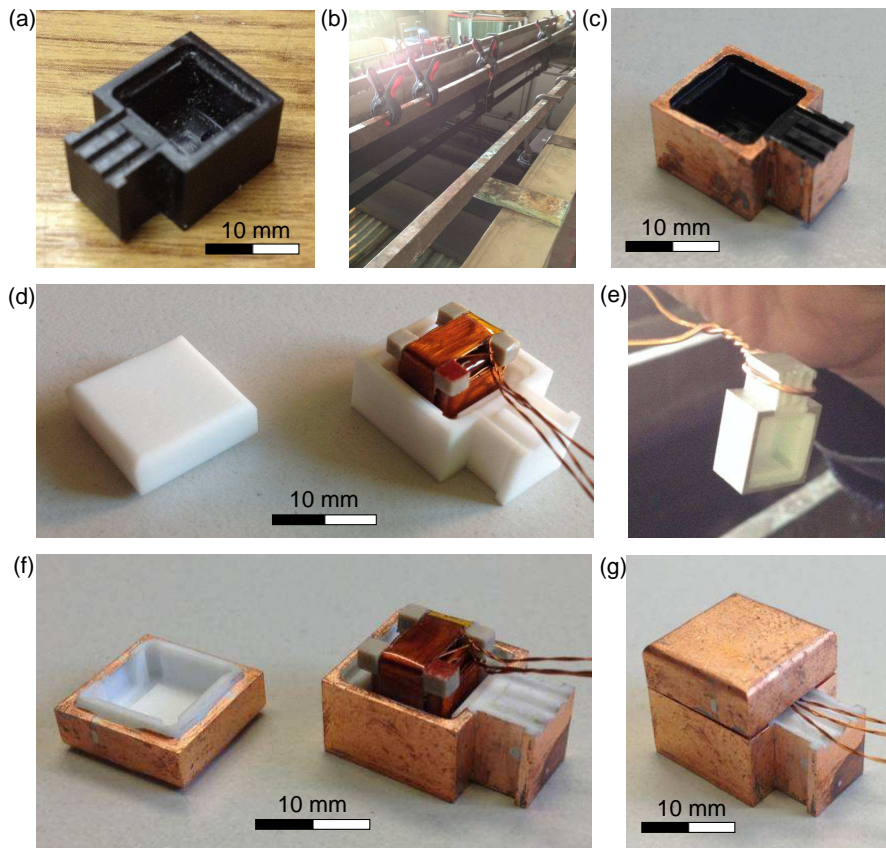

Fig. 4. Manufacturing of a sample of the electrostatic shield: (a) sample 1, made of Delrin, after milling; (b) galvanic bath; (c) sample 1 after coating; (d) sample 2, made of Macor, after milling; (e) sample 2 with silver paint on the surfaces to be coated; (f) sample 2 after coating, open; (g) sample 2 after coating, closed.

- heavy scratching (with a screwdriver) caused some local removal of the electrodeposited layer.

As the electrodeposited layer will be protected by the external case, this result is considered acceptable for the implementation in the HRP diagnostic head.

Summarizing, the tests on thin copper electrodeposition provided the best results for the ceramic materials: Boron Nitride, Alumina and Macor. The latter was selected for the shield support for its mechanical properties, low roughness and for its easier machining.

\section{Construction of Two Samples of the Shields FOR THE MAGNETIC SENSORS}

Due to the complicated shape, small dimensions and extremely thin thickness of the copper layer, the construction of the magnetic sensor shields is not straightforward. Hence two samples of this critical part have been constructed:

- Sample 1: Delrin (polyoxymethylene) sample of the shield base, shown in Fig. 4a

- Sample 2: Macor sample, featuring a shield base and a shield cover, shown in Fig. 4d.

The design of the shields for the magnetic sensors have been developed in order to obtain a design compatible with the limitations of the state-of-the-art milling tools. Due to the very small size of the pieces, the construction procedure have been first tested on Delrin and then extended to Macor. After the construction, the samples have been prepared for electrodeposition by covering the surfaces to be electrodeposited with a silver paint (see Fig. 4e). Then, the pieces have been immersed in a galvanic bath (see Fig. 4b), where an ultra-thin layer of 5 

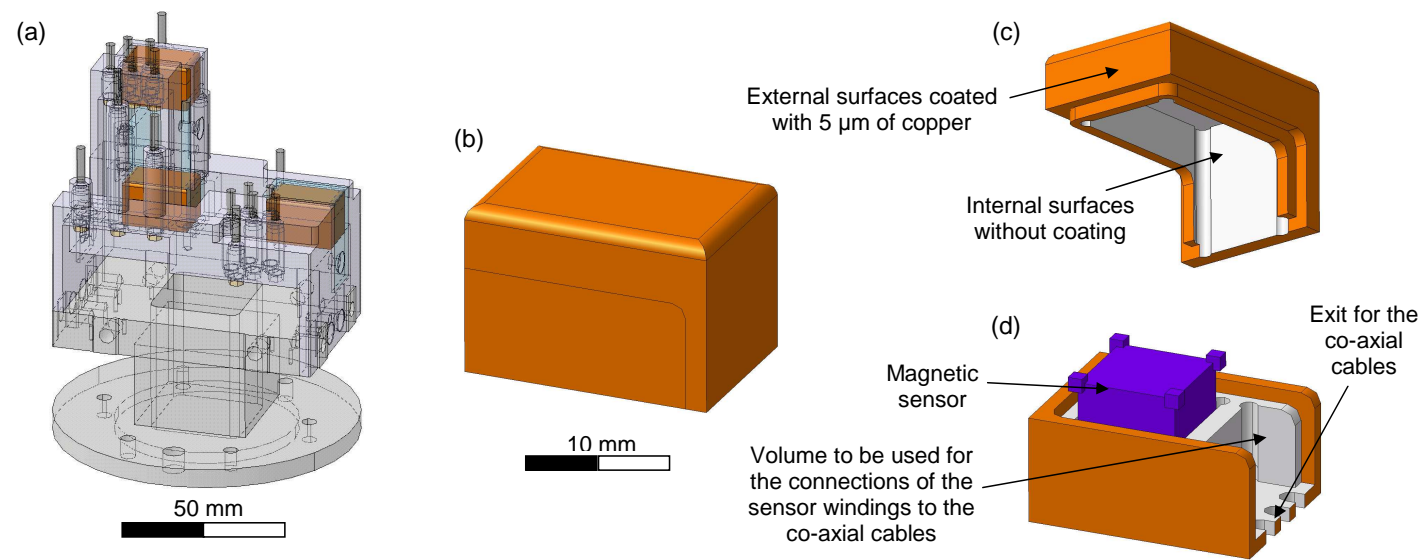

Fig. 5. Design of the electrostatic shields for the magnetic sensors: (a) position of the three magnetic sensors; (b) magnetic sensor shield; (c) cover part; (d) bottom part and magnetic sensor. The parts in orange are coated with a $5 \mu \mathrm{m}$ layer of copper.

$\mu \mathrm{m}$ have been applied (see Fig. $4 \mathrm{c}$ for the Delrin sample and Fig. $4 \mathrm{f}$ and $4 \mathrm{~g}$ for the Macor sample). Following the experience of the previous coating tests, described in the previous section, and of a preliminary test on the Delrin sample, the required thickness of $5 \mu \mathrm{m}$ was obtained on the Macor sample using an electrodeposition voltage of $0.7 \mathrm{~V}$ and a time length in the galvanic bath of 2 minutes.

Sample 2 have been then tested in order to check its effectiveness in shielding the magnetic sensors from the ECRH radiation. The test were successful, hence the manufacturing process was confirmed.

Moreover, during the manufacturing of the samples, there were several discussions on how to optimize various functional and manufacturing aspects of the magnetic sensor shields and as a consequence their design was improved to the one shown in Fig. 5. The main advantages of the updated design are the following:

- the connections of the magnetic sensor to the co-axial cables that will bring out the signals are also shielded;

- the shielding is more uniform, due to the almost cubic shape and to the new position of the holes to let out the cables, that were moved to the edge of the lower surface to avoid the interruption of the shield in a critical zone;

- the manufacturing process is simplified.

\section{CONCLUSION}

The HRP head has been developed to provide information on parallel current density associated to filamentary electromagnetic turbulent structures in the edge region of Wendelstein 7-X experiment. The engineering design of this probe has been developed in order to minimize the volume of the head and maximize its robustness, taking also into account the manufacturing feasibility and the easiness of assembly.

An R\&D campaign has been carried out to choose the most suitable material for the shields for the magnetic sensors. Then, the mechanical feasibility of the shield design has been demonstrated by manufacturing two samples representing the main technological issues. The following tests have demonstrated that the shield is effective in filtering the radiations from ECRH. Moreover, the manufacturing of the samples has permitted to improve the design of this critical part.

\section{ACKNOWLEDGMENT}

This work has been carried out within the framework of the EUROfusion Consortium and has received funding from the Euratom research and training programme 2014-2018 under grant agreement No 633053. The views and opinions expressed herein do not necessarily reflect those of the European Commission.

\section{REFERENCES}

[1] T. Klinger, et al., Performance and properties of the first plasmas of Wendelstein 7-X, Plasma Phys. Control. Fusion 59 (2017) 014018.

[2] T. Sunn Pedersen, et al., Plans for the first plasma operation of Wendelstein 7-X, Nucl. Fusion 55 (2015) 126001.

[3] D. Nicolai, et al., A multi-purpose manipulator system for W7-X as user facility for plasma edge investigation, Fusion Eng. Des. 123 (2017) 960964.

[4] P. Drews, et al., Measurement of the plasma edge profiles using the combined probe on W7-X, Proceedings of 26th IAEA Fusion Energy Conference, Nucl. Fusion 57 (2017) 126020.

[5] R. König, et al., Status of the diagnostics development for the first operation phase of the stellarator Wendelstein 7-X, Rev. Sci. Instrum. 85 (2014) 11D818.

[6] O. Neubauer, et al., Diagnostic setup for investigation of plasma wall interactions at Wendelstein 7-X, Fusion Eng. Des. 96-97 (2015) 915-922.

[7] M. Spolaore, et al., Direct Measurement of Current Filament Structures in a Magnetic-Confinement Fusion Device, Phys. Rev. Lett. 102 (2009) 165001 .

[8] N. Vianello, et al., Drift-Alfvén vortex structures in the edge region of a fusion relevant plasma, Nucl. Fusion 50 (2010) 042002.

[9] I. Furno, et al., Direct Two-Dimensional Measurements of the FieldAligned Current Associated with Plasma Blobs, Phys. Rev. Lett. 106 (2011) 245001

[10] O. Grulke, et al., Analysis of large-scale fluctuation structures in the scrape-off layer of the Wendelstein 7-AS stellarator, Physics of Plasmas 8, 5171 (2001)

[11] M. Spolaore, et al., H-mode achievement and edge features in RFX-mod tokamak operation, Nucl. Fusion 57 (2017) 116039.

[12] Tsui H.Y.W., et al., A new scheme for Langmuir probe measurement of transport and electron temperature fluctuations, Rev. Sci. Instrum. 63 (1992) 4608

[13] J. A. Van Allen, et al., Inference of magnetospheric currents from multipoint magnetic field measurements, Am. J. Phys. 74 (2006) 809.

[14] M. Spolaore, et al., Electromagnetic turbulent structures: A ubiquitous feature of the edge region of toroidal plasma configurations, Physics of Plasmas 22 (2015) 012310. 
[15] K. Kovarik, et al., Filamentary probe on the COMPASS tokamak, Rev. Sc. Instr. 88 (2017) 035106

[16] M. Spolaore, et al., Electromagnetic ELM and inter-ELM filaments detected in the COMPASS Scrape-Off Layer, Nuclear Materials and Energy 12 (2017) 844 http://dx.doi.org/10.1016/j.nme.2016.12.014.

[17] http://www.bn.saint-gobain.com/Combat-Solid-GradeAX05.aspx

[18] D. Hathiramani, et al., Microwave stray radiation: Measures for steady state diagnostics at Wendelstein 7-X, Fusion Eng. Des. 88 (2013) 12321235.

[19] F. M. Tesche, M. Ianoz, T. Karlsson, EMC analysis methods and computational models, John Wiley \& Sons (1997).

[20] P. Agostinetti, et al., Investigation of the Thermo-mechanical Properties of Electro-deposited Copper for ITER, J. Nucl. Mater. 417 (2011) 924 927.

[21] P. Agostinetti, et al., Manufacturing and Testing of Grid Prototypes for the ITER Neutral Beam Injectors, IEEE Trans. on Plasma Science, 42 (2014) 628-632.

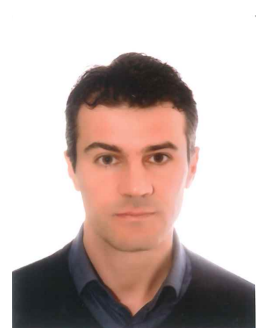

Piero Agostinetti received the Masters degree in Mechanical Engineering from the University of Padova, Italy, in 2003 and the Ph.D. in Energetics from the same University in 2008.

Since 2005 he has been with Consorzio RFX (Padova, Italy). In the framework of EFDA, F4E, EUROfusion and ITER-Organization research programmes, he has been involved in several projects related to the R\&D, construction and operation of neutral beam injectors and diagnostic systems for fusion experiments. In this regard, he has participated to several collaboration projects with IPP (Garching, Germany), NIFS (Toki, Japan), QST (Naka, Japan), CEA (Cadarache, France) and ITER (Cadarache, France).

Since 2010, he is among the technical coordinators of the experimental sessions in the RFX experiment. He has collaborated since 2006 as lecturer with the University of Padova. He has authored or coauthored over 60 papers in peer review journal and over 40 conference proceedings, and is the holder of an Italian and International patent (WO 2013182962).

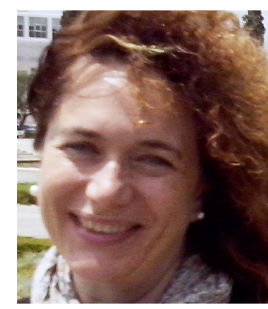

Monica Spolaore obtained the degree in Physics at the Padova University in 1994 and the $\mathrm{PhD}$ in Energetics at the Electrical Engineering Department of the Padova University in 1999. Presently she is a Resercher at the Italian National Research Council, working mainly on the field of edge plasma physics and plasma-wall interaction in fusion devices. She is responsible of the IT group at the Consorzio RFX, Padova. She is co-author of numerous papers published in refereed international journals and coauthor of one European Patent (EP 2249732) and one Italian Patent (N. 001351843)

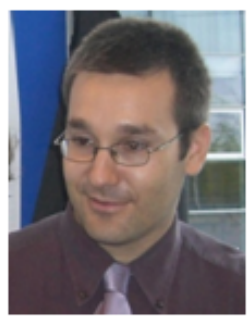

Simone Peruzzo received the Master's and the Ph.D. degrees in electrical engineering from University of Padova, Italy, in 1994 and 1998 respectively. Since 1997 he has been employed by Consorzio RFX, Padova, Italy, performing research and development activities in fusion science and technology, focused in particular on the design and exploitation of magnetic diagnostics for fusion experiments such as RFX, JET and ITER, where he spent several medium term secondments. From 2001 to 2008 he was involved in two JET Enhancement Projects on Magnetic Diagnostics, acting as designer and ultimately also as Project Leader. From 2009 to 2015 he has been involved in design of magnetic diagnostics for ITER, in the framework of several contracts promoted by EFDA, Fusion for Energy and ITER-Organization. Since 2013 he has been Head of the "Plasma Engineering Research Group" of Consorzio RFX. Since 2016 he has been Project Manager of the "Design of machine upgrades for the RFX-mod experiment".

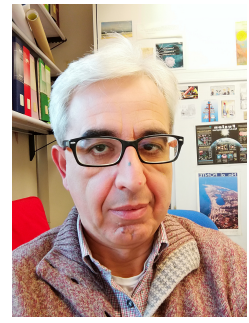

Emilio Martines earned a degree in physics at the University of Pisa, Italy, in 1991 and a Ph.D. in Energetics at the University of Padova, Italy in 1995. Since 1995 he is researcher at the Italian National Research Council (CNR), seconded at Consorzio RFX, in Padova. Since 2007 he is the head of one of the five physics groups operating within Consorzio RFX. His research interests cover fusionrelevant plasmas, with particular emphasis on edge plasma properties, turbulence studies, and helical equilibria in RFP plasmas, and several aspects of plasma applications, with emphasis on plasma medicine. He is author or coauthor of 155 papers published in refereed international journals and of 105 papers published in proceedings of international conferences. The H-index of his scientific production is 30 . He is co-author of two international patents and one European patent, in the fields of plasma medicine and space propulsion. He has been teaching since academic year 2005-2006 the course of Physics of Nuclear Fusion and Plasma Applications within the master's degree in Physics at the University of Padova, and is member of the Academic Council of the Joint Research Doctorate on Fusion Science and Engineering.

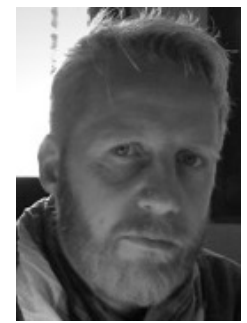

Nicola Vianello received the M.S. in Physics in 1999 from the University of Padova and the PhD in Energetics from the same University in 2002. He worked from 2002 for the Consorzio RFX and since 2009 he is a researcher for the Consiglio Nazionale delle Ricerche (CNR) still working at Consorzio RFX. He has been on leave from the CNR from March 2015 till February 2016 and he spent this year as senior researcher for the Swiss Plasma Center at the Ecole Polytechnique Federale de Lausanne (EPFL). His research interests include turbulence and transport in fusion relevant plasma with experimental work done in a variety of different magnetic configuration (Reversed Field Pinches, Stellarators, Tokamaks). He is the scientific coordinator of experimental activity in different European Tokamaks within the EUROfusion Medium Size Tokamaks framework. He has authored or coauthored over 120 papers in peer review journal and more than 90 conference proceedings. 


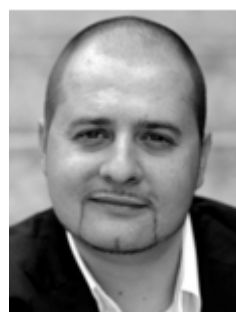

Michele Visentin received the mechanical expert diploma from the State Technical Industrial Institute "F. Viola" of Rovigo, Italy in 2003. From 2008 to 2010 he worked as laboratory professor, teaching mechanical manufacturing processes and engine mechanics at the Professional Institutes of Porto Tolle and Rovigo, Italy, and laboratory of mathematics and physics at the Technical Institute of Rovigo, Italy. Since 2011, he has worked in the drawing office of Consorzio RFX, where he has been involved in the design of the Neutral Beam Test Facility for the ITER neutral beam injectors, and of the MITICA, RFX and Wendelstein 7-X experiments. 